\title{
ON THE STRUCTURE OF GOULDEN-JACKSON-VAKIL FORMULA
}

\author{
S. SHADRIN
}

\begin{abstract}
We study the structure of the Goulden-Jackson-Vakil formula that relates Hurwitz numbers to some conjectural "intersection numbers" on a conjectural family of varieties $X_{g, n}$ of dimension $4 g-3+n$. We give explicit formulas for the properly arranged generating function for these "intersection numbers", and prove that it satisfies Hirota equations. This generalizes and substantially simplifies our earlier results with Zvonkine.
\end{abstract}

\section{Introduction}

1.1. Hurwitz numbers. Consider a ramified covering of degree $d$ over the sphere $\mathbb{C P}^{1}$ by a smooth Riemann surface of genus $g$. Assume that there is a total ramification over $0 \in \mathbb{C P}^{1}$ (that is, the monodromy at 0 is a cycle of length $d$ ); there are $n$ preimages of $\infty \in \mathbb{C P}^{1}$ with the multiplicities $b_{1}, \ldots, b_{n}$; and there is exactly one simple ramification over each of $m=2 g-1+n$ fixed points $z_{1}, \ldots, z_{m} \in \mathbb{C P}^{1} \backslash\{0, \infty\}$ (the number $m$ is determined by the Riemann-Hurwitz formula).

There is a finite number of such coverings. The Hurwitz number $h_{g, b_{1}, \ldots, b_{n}}$ counts these coverings with weights; the weight of a covering equals to the reciprocal of the order of its automorphism group.

1.2. GJV-formula. Goulden, Jackson, and Vakil studied the structure of these Hurwitz numbers in [4]. They observed that these numbers can be represented as

$$
\frac{h_{g, b_{1}, \ldots, b_{n}}}{d \cdot m !}=\int_{X_{g, n}} \frac{1-\lambda_{2}+\cdots \pm \lambda_{2 g}}{\left(1-b_{1} \psi_{1}\right) \cdots\left(1-b_{n} \psi_{n}\right)} .
$$

Here $X_{g, n}$ is a conjectural complex algebraic variety of complex dimension $4 g-$ $3+n$, and $\psi_{1}, \ldots, \psi_{n} \in H^{2}\left(X_{g, n}, \mathbb{C}\right)$ and $\lambda_{2 i} \in H^{4 i}\left(X_{g, n}, \mathbb{C}\right), i=1, \ldots, g$, are some conjectural cohomology classes on it. There is a reasonable hope that $X_{g, n}$ might be something like a suitable compactification of the universal Picard variety; and $\psi$ and $\lambda$-classes might play the same significant role as the usual $\psi$ - and $\lambda$-classes do in geometry of the moduli space of curves.

1.3. Relation to Hirota equations. A consequence of the GJV conjecture is that the "intersection numbers"

$$
\left\langle\lambda_{2 i} \tau_{d_{1}} \cdots \tau_{d_{n}}\right\rangle:=\int_{X_{g, n}} \lambda_{2 i} \psi_{1}^{d_{1}} \cdots \psi_{n}^{d_{n}},
$$

Received by the editors April 17, 2008. 
$4 g-3+n=2 i+d_{1}+\cdots d_{n}$, are not only some important combinatorial constants (defined via Hurwitz numbers and GJV-formula), but they might also be related to geometry and deserve further study.

The first step in this direction was done by me and Zvonkine in [15]. We proved that the generating series for the numbers $\left\langle\tau_{d_{1}} \cdots \tau_{d_{n}}\right\rangle$ is a solution to Hirota hierarchy and to the linearized KP hierarchy simultaneously. This is a complete analogue of the Witten-Kontsevich theorem $[1,7,8,9,10,11,14,16,17]$ for the intersection numbers of $\psi$-classes on the moduli space of curves.

1.4. Kazarian's study of Hodge integrals. In the case of the usual moduli space, there is another formula that relates the intersection number with $\psi$-classes and at most one $\lambda$-class (Hodge integrals) to the combinatorics of ramified coverings. It is the celebrated ELSV-formula [3, 5]. It was recently used by Kazarian [7] in order to give a generalization of the Witten-Kontsevich theorem. He rearranges the generating series for Hodge integrals in such a way that is appears to be a solution of Hirota hierarchy.

In this note we apply the technique proposed by Kazarian in order to expand the structure of GJV-formula. It works perfectly; we also relate the generating series for our "intersection numbers" $\left\langle\lambda_{2 i} \tau_{d_{1}} \cdots \tau_{d_{n}}\right\rangle$ to Hirota equations. Moreover, all computations in this case appear to be much more simple. In particular, we manage to give a very explicit formulas for the generating series of our conjectural "intersection numbers". Of course, we hope that this computation will be helpful for the search of the proper family of varieties $X_{g, n}$ of dimension $4 g-3+n$, whose existence and explicit description is demanded as a part of the Goulden-Jackson-Vakil conjecture.

1.5. Hirota equations. In this paper we call by Hirota equation the bilinear Hirota form of the Kadomtsev-Petviashvili hierarchy. So, a formal power series satisfies Hirota equations if and only if it is a tau-function of the KP hierarchy. For the complete definition and discussion of Hirota equations, we refer the reader to [2, 6, 7 , 12, 15]. For our purposes in this paper, we use a few properties of Hirota equations. That is:

(1) For any $c \in \mathbb{C}$, the function $c+q_{1}$ satisfies Hirota equations.

(2) The operators

$$
\begin{aligned}
& \Lambda_{1}=\sum_{i=2}^{\infty} q_{i} \frac{(i-1) \partial}{\partial q_{i-1}} \\
& \Lambda_{a}=\sum_{i=1}^{\infty} q_{i} \frac{(i-a) \partial}{\partial q_{i-a}}+\frac{1}{2} \sum_{j=1}^{-a-1} \frac{j(-a-j) \partial^{2}}{\partial q_{j} \partial q_{-a-j}}, \quad a \leq 0 \\
& M_{0}=\frac{1}{2} \sum_{i, j=1}^{\infty} q_{i} q_{j} \frac{(i+j) \partial}{\partial q_{i+j}}+\frac{1}{2} \sum_{i, j=1}^{\infty} q_{i+j} \frac{i j \partial^{2}}{\partial q_{i} \partial q_{j}} \\
& M_{1}=\frac{1}{2} \sum_{i+j \geq 1}^{\infty} q_{i} q_{j} \frac{(i+j-1) \partial}{\partial q_{i+j-1}}+\frac{1}{2} \sum_{i, j=1}^{\infty} q_{i+j+1} \frac{i j \partial^{2}}{\partial q_{i} \partial q_{j}}
\end{aligned}
$$




$$
M_{2}=\frac{1}{2} \sum_{i+j \geq 2}^{\infty} q_{i} q_{j} \frac{(i+j-2) \partial}{\partial q_{i+j-2}}+\frac{1}{2} \sum_{i, j=1}^{\infty} q_{i+j+2} \frac{i j \partial^{2}}{\partial q_{i} \partial q_{j}}
$$

are the infinitesimal symmetries of Hirota equations.

(3) Hirota equations are preserved by the rescaling of variables $q_{i} \leftrightarrow u^{i} q_{i}$ (simultaneously for all $i=1,2, \ldots ; u$ is a formal parameter).

Let us give an example of the argument that we use below. Since $c+q_{1}$ satisfies Hirota equations, the series $\exp \left(\Lambda_{1}\right)\left(c+q_{1}\right)=c+\sum_{i=1}^{\infty} q_{i}$ also satisfies Hirota equations.

An important point for us is that if both $f$ and $1+f$ satisfy Hirota equations, it means that $f$ also satisfies the linearized KP hierarchy. So, throughout, we watch for instances where an arbitrary constant may be added to a solution of the Hirota equations.

\section{Rearranged generating series and Hirota equations}

In this section, we study the generating series for all integrals involved in GouldenJackson-Vakil formula.

2.1. Hirota equations and an explicit formula. We define a sequence of linear functions $T_{k}, k=0,1, \ldots$, in formal variables $q_{i}, i=1,2, \ldots$ We set $T_{0}=q_{1}$, and $T_{k+1}=\left(u \Lambda_{0}+\Lambda_{1}\right) T_{k}$. We list the first few expressions:

$$
\begin{aligned}
& T_{0}=q_{1}, \\
& T_{1}=u q_{1}+q_{2}, \\
& T_{2}=u^{2} q_{1}+3 u q_{2}+2 q_{3}, \\
& T_{3}=u^{3} q_{1}+7 u^{2} q_{2}+12 u q_{3}+6 q_{4} .
\end{aligned}
$$

Consider the generating series for the intersection numbers with $\lambda$-classes defined as

$$
G\left(u, q_{1}, q_{2}, \ldots\right)=\sum_{j, k_{1}, k_{2}, \ldots}(-1)^{j}\left\langle\lambda_{2 j} \tau_{0}^{k_{0}} \tau_{1}^{k_{1}} \ldots\right\rangle u^{2 j+1} \frac{T_{0}^{k_{0}}}{k_{0} !} \frac{T_{1}^{k_{1}}}{k_{1} !} \ldots
$$

In particular, we see that the expansion of $G$ in $u$ starts with the terms

$$
G=u F+\frac{u^{2}}{2} \Lambda_{-1} F+\ldots,
$$

where $F$ is the generation function for the intersection numbers without $\lambda$-classes:

$$
F\left(t_{0}, t_{1}, \ldots\right)=\sum_{k_{0}, k_{1}, \ldots}\left\langle\tau_{0}^{k_{0}} \tau_{1}^{k_{1}} \ldots\right\rangle \frac{\left(0 ! q_{1}\right)^{k_{0}}}{k_{0} !} \frac{\left(1 ! q_{2}\right)^{k_{1}}}{k_{1} !} \ldots
$$

Theorem 1. For any function $c=c(u)$, the series

$$
\tau=c(u)+\frac{q_{1}+q_{1} q_{2}}{u}+q_{1}^{2}+\left(\Lambda_{0}+\frac{1}{u} \Lambda_{1}\right)^{2} G\left(u, q_{1}, q_{2}, \ldots\right)
$$

is a solution of the Hirota equations in variables $q_{i}, i=1,2, \ldots$ ( $u$ is just a parameter).

In fact, we can give an explicit formula for $\tau$. 
Theorem 2. We have:

$$
\tau=\exp \left(M_{2}+2 u M_{1}+u^{2} M_{0}\right)\left(c(u)+\frac{q_{1}}{u}\right) .
$$

2.2. Generating series for Hurwitz numbers. Consider the generating series for Hurwitz numbers,

$$
\begin{aligned}
H\left(\beta, p_{1}, p_{2}, \ldots\right) & :=\sum_{g, n} H_{g, n} \\
& :=\sum_{g, n} \frac{1}{n !} \sum_{b_{1}, \ldots, b_{n}} \frac{h_{g, b_{1}, \ldots, b_{n}}}{b \cdot m !} p_{b_{1}} \cdots p_{b_{n}} \beta^{m} .
\end{aligned}
$$

It is proved in [15] (following the observations made before in $[13,8]$ ) that $c(\beta)+\Lambda_{0}^{2} H$ is a solution of the Hirota equations in variables $p_{i}, i=1,2, \ldots$, for any function $c(\beta)$.

For completeness, we remind the Reader of the proof. Hurwitz numbers satisfy a so-called cut-and-join equation (see, e. g. [4]). This implies that $H=\exp \left(\beta M_{0}\right) H_{0,1}$. Since $\left[M_{0}, \Lambda_{0}\right]=0$, we have:

$$
\Lambda_{0}^{2} H=\exp \left(\beta M_{0}\right) \Lambda_{0}^{2} H_{0,1}=\exp \left(\beta M_{0}\right) \sum_{i=1}^{\infty} p_{i} .
$$

Since the series $c(\beta)+\sum_{i=1}^{\infty} p_{i}$ satisfies the Hirota equations, and $M_{0}$ is an infinitesimal symmetry of the Hirota equations, we conclude that $c(\beta)+\Lambda_{0}^{2} H$ also satisfies the Hirota equations.

2.3. Rearranging of GJV-formula. The GJV-formula is applied to all components of $H$ except for $H_{0,1}$ and $H_{0,2}$. Denote by $H_{n}$ the sum $n ! \sum_{g=0}^{n} H_{g, n}$ for $n \geq 3$ and the sum $n ! \sum_{g=1}^{n} H_{g, n}$ for $n=1,2$. That is,

$$
H=\sum_{n=1}^{\infty} \frac{H_{n}}{n !}+H_{0,1}+H_{0,2}
$$

Using that $m=\operatorname{dim} X_{g, n} / 2+n / 2+1 / 2$, we obtain:

$$
\begin{aligned}
H_{n} & =\sum_{g, b_{1}, \ldots, b_{n}} \int_{X_{g, n}} \frac{1-\lambda_{2}+\cdots \pm \lambda_{2 g}}{\left(1-b_{1} \psi_{1}\right) \cdots\left(1-b_{n} \psi_{n}\right)} p_{b_{1}} \cdots p_{b_{n}} \beta^{m} \\
& =\beta \sum_{g, b_{1}, \ldots, b_{n}} \int_{X_{g, n}}\left(1-\beta \lambda_{2}+\cdots \pm \beta^{g} \lambda_{2 g}\right) \prod_{i=1}^{n} \frac{\beta^{1 / 2} p_{b_{i}}}{\left(1-\beta^{1 / 2} b_{i} \psi_{i}\right)} \\
& =\beta\left\langle\left(1-\beta \lambda_{2}+\beta^{2} \lambda_{4}-\ldots\right) \prod_{i=1}^{n}\left(\sum_{d \geq 0} \tau_{d} T_{d}\right)\right\rangle
\end{aligned}
$$

where

$$
T_{d}=\sum_{b \geq 1} \beta^{1 / 2} p_{b} \cdot b^{d} \beta^{d / 2}=\beta^{(d+1) / 2} \sum_{b \geq 1} b^{d} p_{b} .
$$

There is an obvious way to define $T_{d}$ recursively. We just set $T_{0}=\beta^{1 / 2} \sum_{b \geq 1} p_{b}$, and $T_{k+1}=\beta^{1 / 2} \Lambda_{0} T_{k}$. 
2.4. Change of variables. We need the following change of variables. First, we replace $\beta^{1 / 2}$ by $u$. Then we rescale the variables by setting $p_{b}=q_{b} / u^{b}$. And then we replace $q_{i}$ with $\exp \left(-\Lambda_{1} / u\right) q_{i}$.

In other words, it is a linear triangular change of variables given by

$$
p_{b}=\sum_{i=b}^{\infty} \frac{1}{u^{i}}(-1)^{i-b}\left(\begin{array}{l}
i-1 \\
b-1
\end{array}\right) q_{i} .
$$

The same change of variables (in slightly different notation) is used in [15]. Under this change of variable a series $f\left(\beta^{1 / 2}, p_{1}, p_{2}, \ldots\right)$ transforms into $g\left(u, q_{1}, q_{2}, \ldots\right):=$ $\exp \left(-\Lambda_{1} / u\right) f\left(u, q_{1} / u, q_{2} / u^{2}, \ldots\right)$.

Another way to do the same is to replace $p_{b}$ with $\exp \left(-\Lambda_{1}\right) p_{b}$ and then to rescale $p_{b}=q_{b} / u^{b}$. Note that $\Lambda_{1}$ in an infinitesimal symmetry of Hirota equations. The rescaling $p_{b}=q_{b} / u^{b}$ also preserves them. Therefore, this change of variables preserves the property for being a solution to Hirota equations.

2.5. Proof of Theorems 1 and 2. We have already observed that $c(\beta)+\Lambda_{0}^{2} H=$ $\exp \left(\beta M_{0}\right)\left(c(\beta)+\sum_{i=1}^{\infty} p_{i}\right)$ is a solution to Hirota equations. Under the change of variables (14), $c(\beta)+\sum_{i=1}^{\infty} p_{i}$ turns into $c(u)+q_{1} / u$ (it is an explicit computation). Since $\left[M_{0}, \Lambda_{1}\right]=2 M_{1},\left[M_{1}, \Lambda_{1}\right]=M_{2}$, and $\left[M_{2}, \Lambda_{1}\right]=0$, the operator $u^{2} M_{0}$ becomes

$$
\exp \left(-\Lambda_{1} / u\right) u^{2} M_{0} \exp \left(\Lambda_{1} / u\right)=u^{2} M_{0}+2 u M_{1}+M_{2} .
$$

This implies that under the change of variables $(14), c(\beta)+\Lambda_{0}^{2} H$ turns into

$$
\tau=\exp \left(M_{2}+2 u M_{1}+u^{2} M_{0}\right)\left(c(u)+\frac{q_{1}}{u}\right) .
$$

On the other hand, under the change of variables (14), $\Lambda_{0}^{2} H_{0,1}$ turns into $q_{1} / u$, $\Lambda_{0}^{2} H_{0,2}$ becomes $q_{1} q_{2} / u+q_{1}^{2}$, and $T_{0}$ turns into $q_{1}$ (all these observations are simple explicit computations). Also, since $\left[\Lambda_{0}, \Lambda_{1}\right]=\Lambda_{1}$, it follows that $\beta^{1 / 2} \Lambda_{0}$ turns into $\exp \left(-\Lambda_{1} / u\right) u \Lambda_{0} \exp \left(\Lambda_{1} / u\right)=u \Lambda_{0}+\Lambda_{1}$.

Therefore, $H_{n}$ becomes $\sum_{j, b_{1}, \ldots, b_{n}}(-1)^{j}\left\langle\lambda_{2 j} \tau_{b_{1}} \cdots \tau_{b_{n}}\right\rangle u^{2 j+1} T_{b_{1}} \cdots T_{b_{n}}$, where $T_{b}$ is defined in variables $q$ as in equation (3).

So, $c(\beta)+\Lambda_{0}^{2} H=c(\beta)+\Lambda_{0}^{2} H_{0,1}+\Lambda_{0}^{2} H_{0,2}+\Lambda_{0}^{2}\left(\sum_{n \geq 1} H_{n} / n !\right)$ becomes $c(u)+$ $q_{1} / u+q_{1} q_{2} / u+q_{1}^{2}+\left(\Lambda_{0}+\Lambda_{1} / u\right)^{2} G$. The last expression must coincide with $\tau$ in equation (16), and this completes the proof of both theorems.

\section{Intersections of $\psi$-classes}

In this section, we study the formal power series

$$
F\left(q_{1}, q_{2}, \ldots\right)=\sum_{k_{0}, k_{1}, \ldots}\left\langle\tau_{0}^{k_{0}} \tau_{1}^{k_{1}} \ldots\right\rangle \frac{\left(0 ! q_{1}\right)^{k_{0}}}{k_{0} !} \frac{\left(1 ! q_{2}\right)^{k_{1}}}{k_{1} !} \ldots
$$


3.1. An explicit formula. Using two different expressions for $\tau$ given by formulas (7) and (8), we see that

$$
\Lambda_{1}^{2} F+q_{1}+q_{1} q_{2}=\exp \left(M_{2}\right) q_{1} .
$$

It was proved in [4] (and we reprove this below) that $F$ satisfies the string equation, that is,

$$
\frac{\partial}{\partial q_{1}} F=\Lambda_{1} F+\frac{q_{1}^{2}}{2}
$$

It implies that

$$
\Lambda_{1}^{2} F+q_{1}+q_{1} q_{2}=\frac{\partial^{2} F}{\partial q_{1}^{2}} .
$$

Thus we prove the following theorem.

Theorem 3. We have:

$$
\frac{\partial^{2}}{\partial q_{1}^{2}} F=\exp \left(M_{2}\right) q_{1}
$$

In particular, an obvious corollary of this explicit formula is Theorem 2 in [15]:

Theorem 4. For any $c \in \mathbb{C}$, the series $c+\partial^{2} F / \partial q_{1}^{2}$ satisfies Hirota equations.

3.2. String equation. Let us prove the string equation (19). Since $\left[\partial / \partial q_{1}, M_{2}\right]=$ $\Lambda_{1}$, and $\Lambda_{1}$ commutes with $M_{2}$, we have:

$$
\frac{\partial \exp \left(M_{2}\right) q_{1}}{\partial q_{1}}=\Lambda_{1} \exp \left(M_{2}\right) q_{1}+1
$$

If we substitue in this formula $\exp \left(M_{2}\right) q_{1}$ with $\Lambda_{1}^{2} F+q_{1}+\Lambda_{1}\left(q_{1}^{2} / 2\right)$ (using equation (18)), and use that $\partial / \partial q_{1}$ commutes with $\Lambda_{1}$, we obtain

$$
\Lambda_{1}^{2}\left(\frac{\partial F}{\partial q_{1}}\right)=\Lambda_{1}^{2}\left(\Lambda_{1} F+\frac{q_{1}^{2}}{2}\right) .
$$

This implies the string equation (19).

3.3. Towards Virasoro constraints. Though we already have a nice closed formula for $\partial^{2} F / \partial q_{1}^{2}$, it is still might be interesting to find an analog of Virasoro contrains for it. Indeed, our goal is to find a family of varieties with the intersection theory controlled by $F$. And we know that in the usual case of the moduli space of curves the geometry of degenerations of curves is intimately related to the existence and the particular form of Virasoro constraints, see [11]. However, a part of the problem is that $F$ is not an exponential generating function; and in our case it does not make any sense to exponentiate it.

We prove a sequence of some strange equations for $\exp \left(M_{2}\right) q_{1}$; and the first two of them are indeed the string equation and the dilaton equation, already proved in [4].

Proposition 5. For any $n \geq 1$,

$$
\frac{n \partial}{\partial q_{n}} \exp \left(M_{2}\right) q_{1}=\Lambda_{2-n} \exp \left(M_{2}\right) q_{1}+\exp \left(M_{2}\right) q_{1}^{n-1} .
$$


3.3.1. Proof. The proof is based on several observations. Denote by ad $y(x)$ the operator $[x, y]$. Then the operator $n \partial / \partial q_{n}$ is equal to the sum

$$
\frac{n \partial}{\partial q_{n}}=\sum_{i=0}^{\infty} \exp \left(-\operatorname{ad} M_{2}\right) \frac{\left(\operatorname{ad} M_{2}\right)^{i}}{i !}\left(\frac{n \partial}{\partial q_{n}}\right)
$$

We show below that among all operators

$$
O_{i}:=\exp \left(-\operatorname{ad} M_{2}\right) \frac{\left(\operatorname{ad} M_{2}\right)^{i}}{i !}\left(\frac{n \partial}{\partial q_{n}}\right)
$$

only $O_{n-1}$ and $O_{n}$ act nontrivially on $\exp \left(M_{2}\right) q_{1}$. Moreover, one can prove that $O_{n-1} \exp \left(M_{2}\right) q_{1}=n \exp \left(M_{2}\right) q_{1}^{n-1}$.

Observe that $n \Lambda_{2-n}=$ ad $M_{2}\left(n \partial / \partial q_{n}\right)$ is also equal to $\sum_{i=1}^{\infty} i O_{i}$. Thus we have:

$$
\begin{aligned}
\frac{n \partial}{\partial q_{n}} \exp \left(M_{2}\right) q_{1} & =n \exp \left(M_{2}\right) q_{1}^{n-1}+O_{n} \exp \left(M_{2}\right) q_{1} \\
\Lambda_{2-n} \exp \left(M_{2}\right) q_{1} & =(n-1) \exp \left(M_{2}\right) q_{1}^{n-1}+O_{n} \exp \left(M_{2}\right) q_{1},
\end{aligned}
$$

and this implies the statement of the theorem.

3.3.2. Action of $O_{i}$. It is a simple observation that

$$
i ! O_{i} \exp \left(M_{2}\right) q_{1}=\exp \left(M_{2}\right)\left(\left(\operatorname{ad} M_{2}\right)^{i}\left(\frac{n \partial}{\partial q_{n}}\right)\right) q_{1} .
$$

Then,

$$
\left(\left(\operatorname{ad} M_{2}\right)^{i}\left(\frac{n \partial}{\partial q_{n}}\right)\right) q_{1}=\left(\left(\operatorname{ad} M_{2}^{l i n}\right)^{i}\left(\frac{n \partial}{\partial q_{n}}\right)\right) q_{1}
$$

where by $M_{2}^{l i n}$ we denote the linear part of $M_{2}$. And then it is a straightforward calculation to show that for $i \geq 1$

$$
\left(\operatorname{ad} M_{2}^{l i n}\right)^{i}\left(\frac{n \partial}{\partial q_{n}}\right)=\prod_{j=0}^{i-1}(n-j) \sum_{k_{1}, \ldots, k_{i}} q_{k_{1}} \cdots q_{k_{i}} \frac{\left(k_{1}+\cdots+k_{i}+n-2 i\right) \partial}{\partial q_{k_{1}+\cdots+k_{i}+n-2 i}}
$$

Since $k_{l} \geq 1, l=1, \ldots, i$, the terms with $\partial / \partial q_{1}$ appear only for $i \geq n-1$. But the coefficient $\prod_{j=0}^{i-1}(n-j)$ vanishes for $i \geq n+1$. So this operator can be applied non-trivially to $q_{1}$ only if $i=n-1$ or $n$. Then it is obvious that for $i=n-1$ it turns $q_{1}$ into $n ! q_{1}^{n-1}$.

\section{Acknowledgements}

The author is grateful to M. Kazarian for helpful remarks. The author was partially supported by the Vidi grant of NWO. 


\section{References}

[1] Lin Chen, Yi Li, Kefeng Liu, Localization, Hurwitz Numbers and the Witten Conjecture, arXiv:math.AG/0609263.

[2] E. Date, M. Kashivara, M. Jimbo, T. Miwa, Transformation groups for soliton equations, Proc. of RIMS Symposium on Non-Linear Integrable Systems, Singapore, World Science Publ. Co., 39-119 (1983).

[3] T. Ekedahl, S. Lando, M. Shapiro, A. Vainshtein. Hurwitz numbers and intersections on moduli spaces of curves, Invent. Math. 146 (2001), no. 2, 297-327.

[4] I. P. Goulden, D. M. Jackson, R. Vakil, Towards the geometry of double Hurwitz numbers, Adv. Math. 198 (2005), no. 1, 43-92.

[5] T. Graber, R. Vakil, Hodge integrals and Hurwitz numbers via virtual localization, Compositio Math. 135 (2003), no. 1, 25-36.

[6] V. Kac, A. Raina, Bombay lectures on highest weight representations of infinite-dimensional Lie algebras, Adv. Ser. Math. Phys., 2, World Scientific, Teaneck, NJ, 1987

[7] M. Kazarian, KP hierarchy for Hodge integrals, Adv. Math. 221 (2009), no. 1, 1-21.

[8] M. Kazarian, S. Lando, An algebro-geometric proof of Witten's conjecture, J. Amer. Math. Soc. 20 (2007), no. 4, 1079-1089.

[9] Y.-S. Kim, K. Liu, A simple proof of Witten conjecture through localization, arXiv:math.AG/0508384.

[10] M. Kontsevich, Intersection theory on the moduli space of curves and the matrix Airy function, Comm. Math. Phys. 147 (1992), no. 1, 1-23.

[11] M. Mirzakhani, Weil-Petersson volumes and intersection theory on the moduli space of curves, J. Amer. Math. Soc. 20 (2007), no. 1, 1-23.

[12] T. Miwa, M. Jimbo, E. Date, Solitons. Differential equations, symmetries, and infinitedimensinal algebras, Cambridge Tracts in Mathematics, 135. Cambridge University Press, Cambridge, 2000.

[13] A. Okounkov, Toda equations for Hurwitz numbers, Math. Res. Lett. 7 (2000), 447-453.

[14] A. Okounkov, R. Pandharipande, Gromov-Witten theory, Hurwitz numbers, and matrix models, I, arXiv:math.AG/0101147.

[15] S. Shadrin, D. Zvonkine, Changes of variables in ELSV-type formulas, Michigan Math. J. 55 (2007), no. 1, 209-228.

[16] E. Verlinde, H. Verlinde, A solution of 2-dimensional topological quantum gravity, Nuclear Phys. B 348, 457-489.

[17] E. Witten. Two dimensional gravity and intersection theory on moduli space, Surveys in Differential Geometry, vol. 1 (1991), 243-310.

Korteweg-de Vries Institute for Mathematics, University of Amsterdam, P. O. Box 94248, 1090 GE Amsterdam, The Netherlands

E-mail address: s.shadrin@uva.nl

Department of Mathematics, Institute of System Research, Nakhimovsky prospekt 361, Moscow 117218, Russia

E-mail address: shadrin@mccme.ru 